\title{
Avaliação da qualidade do cuidado a idosos nos serviços da rede pública de atenção primária à saúde de Porto Alegre, Brasil
}

\author{
Assessing the quality of care for the elderly in services from public primary health care in \\ Porto Alegre, Brazil
}

\author{
La evaluación de la calidad de la atención a los ancianos en los servicios públicos de atención \\ primaria de salud en Porto Alegre, Brasil
}

Elise Botteselle de Oliveira. Universidade Federal do Rio Grande do Sul (UFRGS). Porto Alegre, RS, Brasil. elisebot@gmail.com Mary Clarisse Bozzetti. Universidade Federal do Rio Grande do Sul (UFRGS). Porto Alegre, RS, Brasil. mcbozzetti@yahoo.com.br Lisiane Hauser. Universidade Federal do Rio Grande do Sul (UFRGS). Porto Alegre, RS, Brasil. lisiane.hauser@ymail.com Bruce Bartholow Duncan. Universidade Federal do Rio Grande do Sul (UFRGS). Porto Alegre, RS, Brasil. bbduncan@ufrgs.br Erno Harzheim. Universidade Federal do Rio Grande do Sul (UFRGS). Porto Alegre, RS, Brasil. ernoharz@terra.com.br (Autor correspondente)

\section{Resumo}

Introdução: 0 envelhecimento populacional gera maiores necessidades em saúde e torna-se um desafio para os serviços de saúde. A Atenção Primária à Saúde (APS) tem papel fundamental na promoção do envelhecimento saudável e no controle das multimorbidades. Objetivo: Avaliar a qualidade dos serviços públicos provedores de APS em Porto Alegre-RS, Brasil, na prestação do cuidado aos idosos. Métodos: Estudo transversal de base populacional, realizado em 2007, que entrevistou 212 idosos atendidos nos quatro tipos de serviços públicos da cidade: as unidades Básicas de Saúde, as equipes da Estratégia Saúde da Família, o Serviço de Saúde Comunitária do Grupo Hospitalar Conceição (SSC-GHC) e o Centro de Saúde Escola Murialdo. Resultados: Houve diferença significativa para os atributos Acesso-utilização, Longitudinalidade, Coordenação do cuidado e dos sistemas de informações, e Orientação familiar e comunitária. 0 SSC-GHC apresentou uma prevalência de 55,8\% ( $p<0,001)$ do alto escore geral de APS, números superiores aos demais serviços. 0 escore geral das práticas preventivas mostrou uma diferença significativa quando foram comparados os serviços em relação ao escore geral de APS, com média de 3,9 (IC 95\%=3,60-4,32) entre os idosos que referiram baixo escore e 5,9 (IC 95\%=5,3-6,5) entre os que referiram alto escore. Discussão: Os resultados mostram escores baixos em todos os tipos de serviços. Os serviços com alto escore geral de APS apresentam prevalências maiores de práticas preventivas. Conclusões: Serviços orientados à APS apresentam-se mais efetivos e oferecem melhores cuidados preventivos recomendados para os idosos.

\section{Abstract}

Introduction: The aging population creates a greater demand creates a greater demand for health services and becomes a challenge for the health care system. Primary Health Care (PHC) plays a fundamental role in promoting healthy aging and controlling multi-morbidity. Objective: To assess the quality of care provided to elderly patients in public PHC in Porto Alegre, Rio Grande do Sul state, Brazil. Methods: A cross-sectional population-based study carried out in 2007, with interviews conducted with 212 elderly patients from four types of municipal public services: Primary Health Centers, Family Health Strategy teams, the "Conceição" Hospital Community Health Service (CH/CHS), and the "Murialdo" School Health Center. Results: There were significant differences between the centers investigated regarding the following attributes: Access-utilization, Longitudinality, Care coordination and information systems, and Family and community orientation. The CH/CHS showed prevalence of $55.8 \%(p<0.001)$ for the overall PHC high score - higher figures in comparison with the other services. The overall score of preventive practices showed a significant association with the services to the overall PHC score, with an average of 3.9 (Cl 95\%=3.60-4.32) among the elderly who presented low scores and 5.9 ( $\mathrm{Cl} 95 \%=5.3-6.5)$ among those presenting high scores. Discussion: The results show low scores in all types of services. The services with a high overall PHC score present higher preventive practice prevalence. Conclusions: PHC-oriented services are more effective and offer greater quality of preventive care recommended for the elderly.

Palavras-chave:

Atenção Primária à Saúde Envelhecimento da População Idoso Morbidade Avaliação de Serviços de Saúde

\section{Keywords:}




\section{Resumen}

Introducción: El aumento de la longevidad crea una mayor demanda de servicios de salud y se convierte en un reto para el sistema de atención de salud. La Atención Primaria de Salud (APS) tiene un papel fundamental en la promoción del envejecimiento saludable y en el control de las multimorbilidades. Objetivo: Evaluar la calidad de los proveedores de servicios públicos de APS en Porto Alegre-RS, Brasil, en la atención a los ancianos. Métodos: Estudio transversal de base poblacional, realizado en 2007, que entrevistó a 212 ancianos atendidos en los 4 tipos de servicios públicos de la ciudad: Ios Centros de Salud de Atención Primaria, los equipos de la Estrategia de Salud de la Familia, el Servicio de Salud Comunitaria del Grupo Hospitalario Conceição (SSC/GHC) y el Centro de Salud Escuela Murialdo. Resultados: Hubo diferencias significativas para los atributos Accesibilidad, Longitudinalidad, Coordinación del cuidado y de los sistemas de información, y Orientación familiar y comunitaria. El SSC/GHC mostró una prevalencia del $55,8 \%$ ( $p<0,001$ ) de la puntuación total máxima de APS, números superiores a los de otros servicios. La puntuación total de las prácticas preventivas mostró una diferencia significativa al comparar los servicios con la puntuación total de la APS, con una media de 3,9 (IC 95\%=3,60-4,32) entre los ancianos que presentaron baja puntuación y 5,9 (IC 95\%=5,3-6,5) entre los que presentaron puntuación más alta. Discusión: Los resultados muestran puntuaciones bajas en todos los tipos de servicios. Los servicios con una alta puntuación total de APS tienen una mayor prevalencia de prácticas preventivas. Conclusiones: Servicios orientados a la APS se muestran más eficaces y ofrecen mejores cuidados preventivos para los ancianos.
Palabras clave:

Atención Primaria de Salud

Envejecimiento de la Población

Anciano

Morbilidad

Evaluación de Servicios de Salud

\section{Introdução}

Porto Alegre é a capital brasileira com a maior proporção de idosos, sendo que $15 \%$ da população tem hoje mais de 60 anos. O envelhecimento populacional é uma resposta à mudança de fenômenos demográficos e de saúde, especialmente a queda da fecundidade e da mortalidade, e o aumento da expectativa de vida ${ }^{1-4}$. Com o aumento da longevidade nas últimas décadas, também ocorreu um aumento do número de doenças crônicas, que, nos idosos, tendem a ser múltiplas 5 . Os sistemas de serviços de saúde, que antes lidavam com problemas agudos, hoje precisam atender às necessidades dos idosos, com uma variedade de fatores de risco, condiçôes crônicas e incapacidades, tendo havido acréscimo na demanda pelos serviços ${ }^{6}$.

A Atenção Primária à Saúde (APS) desempenha um papel essencial na promoção do envelhecimento saudável, já que está ativamente envolvida na gestáo da saúde dos idosos, no diagnóstico e no manejo de suas multimorbidades e na prevenção de fatores de risco para doenças crônicas 7 . O cuidado a idosos representa cerca de 40 a $50 \%$ da atividade total do médico de família e comunidade (MFC) e mais de 50\% das prescriçôes em APS, sendo 75\% destas usadas para tratamento ou prevenção de doenças crônicas de forma contínua ${ }^{8}$. A APS pode ser vista como uma estratégia para fortalecer os sistemas de saúde que visa a aprimorar a efetividade dos serviços e garantir a equidade na sua oferta?

Starfield ${ }^{9}$ sistematizou as características próprias da APS e seus componentes fundamentais e criou uma definição operacional, possibilitando estabelecer o grau de orientação à APS, mediante a presença e a extensão de seus atributos, tanto essenciais como derivados. Os atributos essenciais abordam: a) o papel da APS como a porta de entrada para o sistema de saúde, exceto em situações de emergência (acesso de primeiro contato); b) a manutenção de vínculo pessoal e continuado do paciente com um profissional ou um serviço de saúde (longitudinalidade); c) a capacidade de disponibilizar uma ampla gama de serviços e de resolver problemas (integralidade); e d) o atendimento dos problemas tratados em outros serviços (coordenação). Os atributos derivados abordam a medida com que a APS se concentra na saúde dos indivíduos no contexto da família (orientação familiar), no enfrentamento dos problemas de saúde da comunidade (orientação comunitária) e nos relacionamentos com pessoas de diferentes grupos e culturas (competência cultural) ${ }^{9-11}$. A mesma autora criou o Primary Care Assessment Tool (PCATool), uma ferramenta que avalia aspectos da estrutura e do processo dos serviços de APS, baseada no conceito dos atributos da APS, por meio da coleta de informaçóes do ponto de vista do usuário, do profissional e do gestor dos serviços ${ }^{9,11}$.

A natureza longitudinal do cuidado em APS proporciona múltiplas oportunidades para a realização de intervenções que tenham demonstrado sua capacidade para reduzir a morbidade, melhorando a qualidade de vida dos idosos ou evitando morbidades prematuras ${ }^{12}$. Ultimamente, tem-se dado maior importância às atividades preventivas e de promoção da saúde, na busca de formas mais efetivas e menos dispendiosas de cuidado e, por consequência, de avaliaçóes de custo e de resultados. Estudos recentes demonstram que a adoção de um estilo de vida saudável pode prevenir $60 \%$ da mortalidade prematura e acrescentar 14 anos à expectativa de vida ${ }^{13}$. Apesar da evidência forte da efetividade das práticas preventivas, o seu uso permanece ainda muito baixo; nos EUA, por exemplo, somente $28 \%$ dos fumantes recebem orientaçóes ou tratamento para cessação do tabagismo ${ }^{14}$.

No momento do estudo, a Rede Pública de APS em Porto Alegre possuía quatro tipos de serviços distintos: as Unidades Básicas de Saúde (UBS), com modelo tradicional de atendimento, que atendiam 800 mil pessoas; as unidades com equipes 
da Estratégia Saúde da Família (ESF), implantadas na cidade desde 1996, que apresentavam cobertura de 15,6\% e atendiam mais de 200 mil pessoas; as 12 unidades do Serviço de Saúde Comunitária do Grupo Hospitalar Conceição (SSC-GHC), instaladas na zona norte da cidade desde a década de 1980, que eram responsáveis por mais de 120 mil pessoas, e as sete unidades do Centro de Saúde Escola Murialdo (CSEM), que cobriam em torno de 60 mil pessoas, com início da sua implantação em 1974, na zona leste da cidade, tendo sido municipalizadas recentemente. Tanto o SSC-GHC quanto o CSEM são historicamente centros de saúde-escola formadores de recursos humanos para a APS, com residência multiprofissional e de medicina de família e comunidade, com início recente da incorporação de agentes comunitários de saúde (ACS) às suas equipes. Devido à existência de outros modelos de APS em Porto Alegre, anteriores à criaçáo da ESF - que se mantêm atuando concomitantemente - torna-se importante estudar o papel dos diferentes serviços de saúde da cidade.

Sáo poucos os estudos que avaliam o impacto da APS na saúde dos idosos e sua resposta às dificuldades e aos desafios gerados pelo envelhecimento populacional. Frente a essa lacuna, este estudo tem como objetivo avaliar a qualidade dos diferentes serviços públicos provedores de APS em Porto Alegre na prestação do cuidado aos idosos e a sua associação com a realização de práticas preventivas baseadas em evidências.

\section{Métodos}

Os dados utilizados neste trabalho foram provenientes do estudo transversal de base populacional intitulado "Avaliação da qualidade do processo de atenção e da sua efetividade sobre a saúde do adulto no Programa Saúde da Família e em modelos alternativos no município de Porto Alegre", realizado no período de julho de 2006 a agosto de $2007^{15}$.

A amostragem foi feita por conglomerado de serviços, estratificada por distrito de saúde e proporcional à populaçáo adscrita a cada serviço. Os domicílios foram selecionados de forma sistemática para cada unidade, tendo-se estudado 50\% das unidades de UBS e ESF, e a totalidade das unidades do SSC-GHC e do CSEM. O critério de adscriçáo dos usuários dos serviços públicos de APS foi utilizado apenas para a seleção dos participantes, avaliando-se os serviços de saúde referidos como de uso preferencial pelos entrevistados.

Os indivíduos que aceitaram livremente participar da pesquisa foram entrevistados em seus domicílios por entrevistadores devidamente treinados. Dentre os 3014 entrevistados no projeto, foram considerados para o presente estudo os indivíduos maiores de 60 anos e residentes nas áreas geográficas cobertas pela rede pública de APS.

Foram aplicados o Instrumento de Avaliação da APS (PCATool-Brasil), ${ }^{15}$ um questionário sobre satisfação com serviço de saúde ${ }^{16}$, e um conjunto de perguntas estruturadas sobre variáveis socioeconômicas e demográficas, fatores de risco cardiovasculares, uso de medicamentos, processo de atenção (orientaçôes preventivas, vacinação) e avaliação de saúde. Além disso, foram realizadas medidas antropométricas, incluindo peso, altura, circunferência do quadril e da cintura, e pressão arterial. Os dados foram apropriados por meio de digitalização e verificação com o software Teleform . $^{\oplus}$

O instrumento validado PCATool-Brasil mede a presença e a extensão dos quatro atributos essenciais (acesso de primeiro contato, longitudinalidade, integralidade e coordenação) e de dois atributos derivados (orientação familiar e orientação comunitária) da APS, e o grau de afiliação do usuário ao serviço de saúde ${ }^{10}$. Esse instrumento possibilita, por meio de respostas tipo Likert ( $4=$ com certeza, sim; 3 = provavelmente, sim; $2=$ provavelmente, não; e $1=$ com certeza, não), construir escores de APS com intervalo de 1 a 4 para cada atributo, que são posteriormente transformados em uma escala de 0 a 10. O escore final de cada um desses atributos é formado pela média das respostas de seus itens (perguntas). A média dos escores de todos os atributos e seus componentes produz o escore geral de APS. Esse escore permite agrupar os serviços de saúde em alto grau de orientação à APS quando assume valores maiores ou iguais a 6,7 e, em baixo grau, quando assume valores menores de $6,7^{10}$.

O escore de práticas preventivas foi construído com o objetivo de ser uma medida-resumo das atividades preventivas que devem ser realizadas em idosos. A prevalência das práticas preventivas pertinentes a cada subgrupo foi obtida por respostas dicotômicas $(0=$ ausência e 1 =presença). A partir dessas variáveis, obteve-se o escore médio das práticas preventivas realizadas em cada subgrupo, posteriormente transformadas para que o escore assumisse valores de 0 a 10 . Esse escore foi utilizado para comparar a qualidade do cuidado prestado aos idosos entre os serviços com alto e baixo escore de APS.

Os escores médios de cada atributo da APS por tipo de serviço foram estimados pela metodologia de modelo misto, que permite considerar a estrutura da amostra, na qual as unidades de saúde estão aninhadas ao tipo de serviço ${ }^{17}$. Nesse modelo, 
o tipo de serviço de APS, que representa as categorias sobre as quais se deseja fazer inferência, foi considerado como efeito fixo e as unidades de saúde, que constituem uma amostra das unidades de saúde estudadas, foram consideradas como efeito aleatório. Dessa maneira, as estimativas para o tipo de serviço contemplam a variabilidade dentro das unidades de saúde e também entre essas unidades. Além disso, ao contemplar efeitos aleatórios, é possível inferir os resultados para a população ${ }^{18}$.

No caso da suposição de dados com distribuição normal não satisfeita (coordenação - sistema de informação e orientação familiar), optou-se pela classe de modelos lineares generalizados, na qual são permitidos os ajustes de efeito fixo com dados que não sejam de distribuição normal. Esses modelos, ao serem estendidos para o ajuste de modelos mistos, são chamados modelos lineares generalizados mistos e também permitem incorporar efeitos aleatórios ${ }^{19}$. Para a comparação dos escores médios de APS entre os quatro serviços avaliados, foi utilizado o teste de comparação múltipla de Tukey. Essas análises foram realizadas no software SAS 9.1, utilizando-se os procedimentos Mixed e Glimmix ${ }^{20}$.

$\mathrm{Na}$ comparação de médias, utilizou-se o teste $t$ de Student para amostras independentes. No caso da comparação entre proporçôes, foi utilizado o teste de comparação para proporçôes associado à estatística $\chi^{2}$. Essas análises foram realizadas no pacote estatístico STATA 9.0, levando-se em consideração a estratégia de amostragem (clusters) através do método de linearização de Taylor ${ }^{21}$. Dessa maneira, puderam-se incorporar correçôes nas estimativas de variabilidade, permitindo a adequação da análise ao plano amostral. Foi adotado o nível de significância de 5\% para todas as análises estatísticas.

O projeto desse estudo foi submetido aos Comitês de Ética em Pesquisa da Universidade Federal do Rio Grande do Sul, do Hospital de Clínicas Porto Alegre, do Grupo Hospitalar Conceição, da Escola de Saúde Pública-RS e da Secretaria Municipal de Saúde de Porto Alegre, tendo sido aprovado por todos. Os entrevistados assinaram o Termo de Consentimento Livre e Esclarecido (TCLE) e foi mantida a confidencialidade dos dados coletados. Maiores detalhes sobre a metodologia do estudo inicial podem ser consultados na publicação de seu protocolo de pesquisa, intitulado "Quality and effectiveness of different approaches to primary care delivery in Brazil'15.

\section{Resultados}

Dentre os 3014 usuários entrevistados no estudo inicial, foram identificados 212 idosos (Figura 1), distribuídos entre os serviços públicos de APS da seguinte maneira: 62 nas UBS, 75 nas equipes da ESF, 32 nos postos do CSEM e 43 nas unidades do SSC-GHC. Houve um predomínio de mulheres $(59,9 \%)$, da cor branca $(67,9 \%)$, com companheiro $(60,38 \%)$ e das classes sociais C, D e E (74,5\%). Com relação ao estado de saúde, a prevalência de hipertensão arterial foi maior em UBS $(74,2 \%)$ e a prevalência de diabéticos foi de 25\% e 30,2\% no CSEM e no SSC-GHC, respectivamente (Tabela 1).

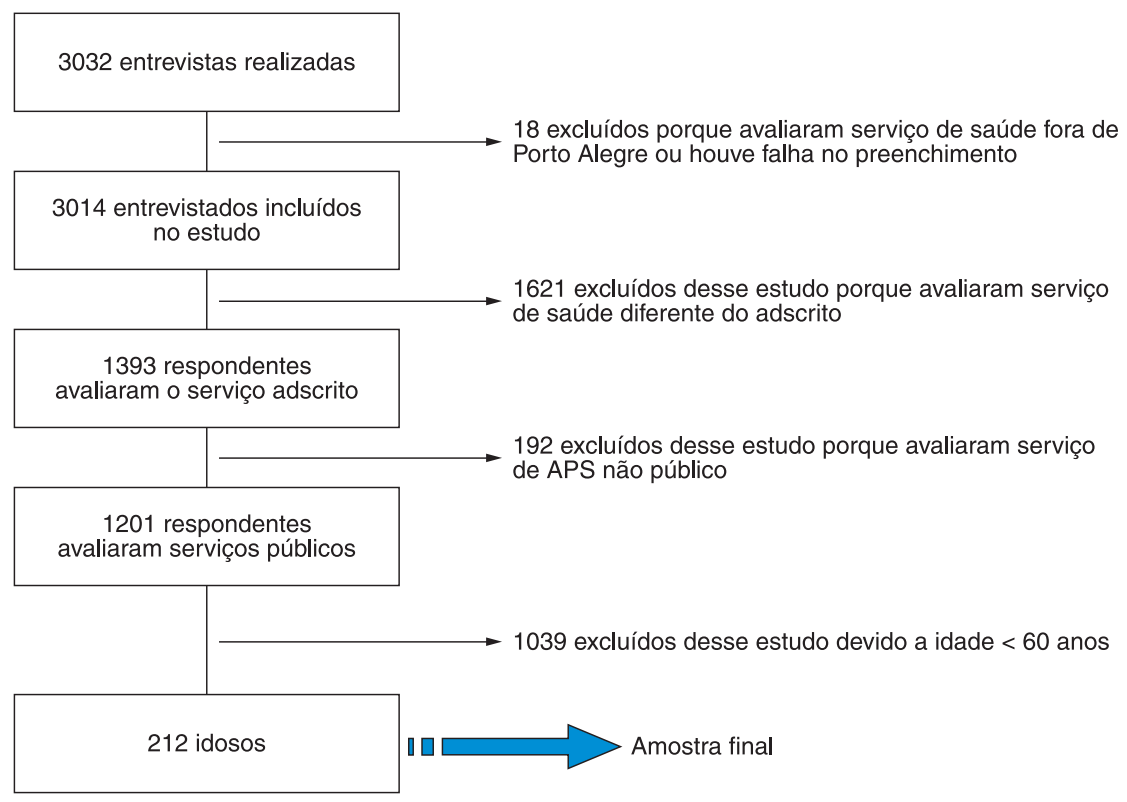

Figura 1. Descrição do processo de amostragem do estudo. Porto Alegre, 2007. 
Tabela 1. Características da população maior de 60 anos usuária dos serviços de APS, Porto Alegre, 2007.

\begin{tabular}{|c|c|c|c|c|c|}
\hline Variáveis & Total & $\begin{array}{l}\text { Unidade Básica de } \\
\text { Saúde (UBS) }\end{array}$ & $\begin{array}{c}\text { Estratégia Saúde da } \\
\text { Família (ESF) }\end{array}$ & $\begin{array}{l}\text { Centro de Saúde } \\
\text { Escola Murialdo } \\
\text { (CSEM) }\end{array}$ & $\begin{array}{c}\text { Serviço de Saúde } \\
\text { Comunitária do Grupo } \\
\text { Hospitalar Conceição } \\
\text { (SSC-GHC) }\end{array}$ \\
\hline & [Média (DP)/\% (n)] & [Média (DP)/\% (n)] & [Média (DP)/\% (n)] & [Média (DP)/\% (n)] & [Média (DP)/\% (n)] \\
\hline & $\mathrm{n}=212$ & $\mathrm{n}=62$ & $n=75$ & $n=32$ & $n=43$ \\
\hline Idade (anos) & $68,24(7,25)$ & $68,74(7,89)$ & $67,85(6,75)$ & $66,84(6,48)$ & $69,23(7,69)$ \\
\hline \multicolumn{6}{|l|}{ Sexo } \\
\hline Masculino & $40,09(85)$ & $48,39(30)$ & $41,33(31)$ & $37,50(12)$ & 27,91 (12) \\
\hline Feminino & $59,91(127)$ & $51,61(32)$ & $58,67(44)$ & $62,50(20)$ & $72,09(31)$ \\
\hline Escolaridade (anos) & $5,04(3,70)$ & $5,45(3,74)$ & $4,22(3,56)$ & $5,59(4,06)$ & $5,48(3,46)$ \\
\hline Renda Familiar (reais) & $1466,50(1246,03)$ & $1535,29(949,60)$ & $1589,23(1633,97)$ & $1175,56(891,24)$ & $1372(1045,59)$ \\
\hline Renda per capita (reais) & $541,84(427,85)$ & $602,77(406,03)$ & $564,04(483,44)$ & $502,24(451,20)$ & $444,38(316,21)$ \\
\hline \multicolumn{6}{|l|}{ Cor da pele } \\
\hline Brancos & $67,92(100)$ & $74,19(46)$ & $57,33(43)$ & $62,50(20)$ & $81,40(35)$ \\
\hline Não brancos & $32,08(68)$ & $25,81(16)$ & 42,67 (32) & $37,50(12)$ & 18,60 (8) \\
\hline \multicolumn{6}{|l|}{ Classe social } \\
\hline$A B$ & $54(25,47)$ & $27,42(17)$ & 22,67 (17) & $31,25(10)$ & $23,26(10)$ \\
\hline CDE & $74,53(158)$ & $72,58(45)$ & $77,33(58)$ & $68,75(22)$ & $76,74(33)$ \\
\hline \multicolumn{6}{|l|}{ Estado marital } \\
\hline sem companheiro & $39,62(84)$ & $45,16(28)$ & $36,00(27)$ & $37,50(12)$ & $39,53(17)$ \\
\hline com companheiro & $60,38(128)$ & $54,84(34)$ & $64,00(48)$ & $62,50(20)$ & $60,47(26)$ \\
\hline Sedentarismo & $35,55(75)$ & $37,10(23)$ & $41,33(31)$ & $15,63(5)$ & $38,10(16)$ \\
\hline DM & $20,75(44)$ & $16,13(10)$ & $17,33(13)$ & $25,00(8)$ & $30,23(13)$ \\
\hline HAS & $72,64(154)$ & $74,19(46)$ & $72,00(54)$ & $71,88(23)$ & $72,09(31)$ \\
\hline Outras doenças crônicas & $79,72(169)$ & $74,19(46)$ & $79,73(59)$ & $96,77(30)$ & $80,95(34)$ \\
\hline Obesidade & $32,38(68)$ & $27,87(17)$ & $32,00(24)$ & $41,94(13)$ & $32,56(14)$ \\
\hline Tabagismo & $18,1(38)$ & $18,03(11)$ & $22,97(17)$ & $21,88(7)$ & $6,97(3)$ \\
\hline Hospitalizações nos últimos 12 meses & $11,32(24)$ & $8,06(5)$ & $10,67(8)$ & $15,63(5)$ & $13,95(6)$ \\
\hline \multicolumn{6}{|l|}{ Saúde autorreferida } \\
\hline boa, muito boa & $66,98(142)$ & $66,13(41)$ & $69,33(52)$ & $71,88(23)$ & $60,47(26)$ \\
\hline regular, ruim, muito ruim & $33,02(70)$ & $33,87(21)$ & $30,67(23)$ & $28,13(9)$ & $39,53(17)$ \\
\hline \multicolumn{6}{|l|}{ Vínculo com o serviço } \\
\hline$\leq 2$ anos & $20,87(43)$ & $22,95(14)$ & $31,08(23)$ & $13,30(4)$ & $4,87(2)$ \\
\hline$>$ 2anos & $79,13(163)$ & $77,05(47)$ & $68,92(51)$ & $86,67(26)$ & $95,12(39)$ \\
\hline \multicolumn{6}{|l|}{ Consultas nos últimos 12 meses } \\
\hline$<2$ & $26,54(56)$ & 30,65 (19) & $21,62(16)$ & $37,50(12)$ & $20,93(9)$ \\
\hline$\geq 2$ & $73,46(155)$ & $69,35(43)$ & $78,38(58)$ & $62,50(20)$ & $79,07(34)$ \\
\hline Ter médico de referência & $34,40(73)$ & $30,60(19)$ & $36,50(27)$ & $9,40(3)$ & $55,80(24)$ \\
\hline
\end{tabular}

A presença de duas ou mais doenças crônicas chegou a 96,8\% no CSEM. Já o estado de saúde autorreferido como bom ou muito bom foi de 69,3\% na ESF e 71,9\% no CSEM. Na ESF e no CSEM, 22,07\% e 21,88\% dos entrevistados, respectivamente, referiram ser tabagistas. O vínculo com o serviço foi maior no SSC-GHC, em que 95,1\% dos entrevistados referiram ser atendidos havia mais de dois anos nesse serviço. No SSC-GHC, 55,8\% referiram ter um médico de referência. Em relação a ter se consultado nos últimos 12 meses anteriores à pesquisa, a população atendida no SSC-GHC foi maior $(79,7 \%)$, seguida da população atendida na ESF (78,3\%) (Tabela 1). 
A comparação do grau de orientação da APS entre os idosos dos diferentes serviços de APS foi significativa para os seguintes atributos: acesso-utilização, longitudinalidade, coordenação do cuidado, coordenação dos sistemas de informações, orientação familiar e orientação comunitária. O mesmo ocorreu para os escores essencial e geral, sendo o SSC-GHC superior aos demais, em comparação com a unidade de referência (UBS) (Tabela 2).

Tabela 2. Comparação dos escores médios dos atributos e frequência do alto escore de APS entre os serviços de APS, Porto Alegre, 2007.

\begin{tabular}{|c|c|c|c|c|c|}
\hline \multirow[t]{7}{*}{ Atributos da APS } & \multicolumn{5}{|c|}{ Escore Médio dos atributos da APS entre os serviços } \\
\hline & Unidade Básica de & Estratégia Saúde da & Centro de Saúde & Serviço de Saúde & valor-p* \\
\hline & Saúde (UBS) & Família (ESF) & Escola Murialdo & Comunitária do Grupo & \\
\hline & & & (CSEM) & Hospitalar Conceição & \\
\hline & & & & (SSC-GHC) & \\
\hline & Média (IC 95\%) & Média (IC 95\%) & Média (IC 95\%) & Média (IC 95\%) & \\
\hline & $\mathrm{n}=62$ & $\mathrm{n}=75$ & $n=32$ & $\mathrm{n}=43$ & \\
\hline Acessibilidade & $4,10(3,40-4,70)$ & $3,50(3,00-4,10)$ & $3,30(2,30-4,30)$ & $3,70(2,90-4,50)$ & 0,500 \\
\hline Acesso - utilização & $8,50(8,10-8,90)$ & $9,00(8,60-9,30)$ & $9,50(8,90-10,00)^{\S}$ & $9,60(9,10-10,00)^{\S}$ & 0,005 \\
\hline Longitudinalidade & $6,20(5,60-6,80)$ & $7,10(6,50-7,60)$ & $6,20(5,30-7,10)$ & $7,80(7,10-8,50)^{\S}$ & 0,001 \\
\hline Coordenação do cuidado & $3,20(2,30-4,20)$ & $6,00(5,10-6,90)^{\S}$ & $4,40(2,90-5,90)$ & $6,50(5,30-7,60)^{\S}$ & $<0,001$ \\
\hline Coordenação - sistema de informações & $5,60(5,20-6,00)$ & $6,30(5,80-6,80)$ & $5,10(5,40-6,60)$ & $6,97(6,30-7,60)^{\S}$ & 0,005 \\
\hline Integralidade - serviços disponíveis & $5,50(5,00-6,10)$ & $4,80(4,30-5,30)$ & $5,60(4,70-6,50)$ & $6,10(5,40-6,80)^{z}$ & 0,030 \\
\hline Integralidade - serviços prestados & $3,30(2,50-4,10)$ & $3,50(2,80-4,20)$ & $3,50(2,30-4,60)$ & $4,50(3,60-5,40)$ & 0,120 \\
\hline Orientação familiar & $3,30(2,40-4,20)$ & $3,90(3,10-4,70)$ & $4,70(3,40-5,20)$ & $6,10(5,10-7,20)^{8 z}$ & $<0,001$ \\
\hline Orientação comunitária & $2,30(1,40-3,30)$ & $5,90(5,10-6,70)^{\S}$ & $4,70(3,30-6,00)^{\S}$ & $7,20(6,10-8,30)^{\S}$ & $<0,001$ \\
\hline Escore Essencial & $5,50(5,10-5,90)$ & $5,90(5,50-6,20)$ & $5,70(5,10-6,20)$ & $6,70(6,30-7,20)^{\text {§z\& }}$ & $<0,001$ \\
\hline Escore Geral & $5,00(4,62-5,46)$ & $5,70(5,30-6,00)$ & $5,40(4,80-6,10)$ & $6,70(6,20-7,20)^{\S \&}$ & $<0,001$ \\
\hline \multirow[t]{3}{*}{ Atributos da APS } & \multicolumn{5}{|c|}{ Frequência de Alto Escore da APS nos serviços } \\
\hline & $\mathrm{n}=62$ & $\mathrm{n}=75$ & $\mathrm{n}=32$ & $\mathrm{n}=43$ & valor-p \\
\hline & {$[\%](n)$} & {$[\%](n)$} & {$[\%](n)$} & {$[\%](n)$} & \\
\hline Escore Essencial & $20,97(13)$ & $32,00(24)$ & $25,00(8)$ & $55,81(24)$ & $<0,001^{\star *}$ \\
\hline Escore Geral & 19,35 (12) & $26,67(20)$ & $21,88(7)$ & $55,81(24)$ & $<0,001^{\star \star}$ \\
\hline
\end{tabular}

"Os escores assumem valores entre 0 e 10; alto grau de orientação à APS, quando escore $\geq 6,7$. *Significância associada ao teste de comparação de médias pela Análise de Variância (ANOVA): valor- $p<0,05$. §Significância pelo teste de Tukey; em comparação à UBS: valor- $p<0,05$. ${ }^{3}$ Significância pelo teste de Tukey; em comparação à ESF: valor- $p<0,05$. ${ }^{~}$ Significância pelo teste de Tukey; em comparação ao CSEM: valor- $p<0,05$. ** Significância associada ao teste de comparação de proporções utilizando a estatística $\chi^{2}$ : valor- $p<0,05$.

O SSC-GHC também apresentou diferença para integralidade - serviços disponíveis e orientação familiar - em relação à ESF, para orientação comunitária em comparação com o CSEM, para o escore essencial em relação à ESF e ao CSEM, e no escore geral também em relação à ESF e ao CSEM. O escore geral da APS também foi significativamente diferente entre os três serviços avaliados, sendo o serviço base de comparação, a UBS (Tabela 2). Na Tabela 2, na qual também é apresentada a frequência de alto escore entre estes serviços, o SSC-GHC apresentou uma prevalência de $55,8 \%$ de alto escore essencial e 55,8\% de alto escore geral, números superiores aos demais serviços em relação à presença dos atributos da APS.

Em referência ao processo de atençấo e à qualidade do cuidado dos idosos, a Tabela 3 apresenta diferenças entre aqueles atendidos em serviços com al to e baixo grau de orientaçáo à APS. São significativas e superiores naqueles atendidos em serviços com alto escore as orientaçóes de atividade física, dieta saudável e higiene bucal, para a população geral; orientação para cuidado dos pés, encaminhamento para dentista e uso de metformina, para os pacientes portadores de diabetes; solicitaçáo de exame de urina e perfil lipídico, e medida da altura para os pacientes portadores de mais de uma doença crônica. Além disso, a medida de satisfação com a última consulta também foi melhor nos serviços com alto escore. As demais medidas de resultado não mostraram diferenças significativas. $\mathrm{O}$ escore geral das práticas preventivas mostrou uma diferença significativa entre os serviços avaliados, com média de 3,9 entre os idosos que referiram baixo Escore Geral de APS, e de 5,9 entre os que referiram utilizar serviços com alto Escore Geral de APS (Tabela 3). 
Tabela 3. Indicadores da qualidade do cuidado de idosos em relação ao escore geral de APS, Porto Alegre, 2007.

\begin{tabular}{|c|c|c|c|c|}
\hline Práticas preventivas & $\mathrm{n}=212$ & $\begin{array}{l}\text { Baixo Escore Geral de } \\
\text { APS } n=159 \\
{[\%](n)}\end{array}$ & $\begin{array}{l}\text { Alto Escore Geral de } \\
\qquad \text { APS } n=53 \\
{[\%](n)}\end{array}$ & valor-p \\
\hline \multicolumn{5}{|l|}{ Medidas de processo } \\
\hline Orientação para cessar o hábito de fumar & 41 & $50,00(32)$ & $66,70(9)$ & 0,270 \\
\hline Orientação para diminuir ou parar de beber & 73 & $7,40(56)$ & $21,00(17)$ & 0,130 \\
\hline Orientação para realização de exercícios & 208 & $34,00(157)$ & $55,70(51)$ & $0,004^{*}$ \\
\hline Orientação de dieta saudável & 208 & $49,00(157)$ & $78,70(51)$ & $<0,001^{*}$ \\
\hline Orientação sobre higiene bucal & 207 & $10,20(157)$ & $58,30(50)$ & $<0,001^{*}$ \\
\hline Pressão arterial aferida no último ano & 208 & $75,50(157)$ & $86,70(51)$ & 0,050 \\
\hline Realização de vacina contra influenza & 208 & $59,20(157)$ & $70,50(51)$ & 0,180 \\
\hline Encaminhamento para oftalmologista no último ano & 43 & $23,10(28)$ & $47,10(15)$ & 0,100 \\
\hline Encaminhamento para dentista no último ano & 44 & $3,70(29)$ & $29,40(15)$ & $0,020^{*}$ \\
\hline Exame dos pés realizado no último ano & 44 & $7,40(29)$ & $41,20(15)$ & $0,020^{*}$ \\
\hline Orientação para cuidado dos pés & 44 & $29,60(29)$ & $58,80(15)$ & $0,010^{*}$ \\
\hline Participa de grupo de educação para diabéticos & 44 & $14,80(29)$ & $23,50(15)$ & 0,440 \\
\hline Uso de metformina & 151 & $11,40(114)$ & $27,00(37)$ & $0,006^{*}$ \\
\hline Participação grupo de educação para hipertensos & 117 & $11,40(84)$ & $21,00(33)$ & 0,250 \\
\hline Uso de tiazídicos & 151 & $53,40(114)$ & $53,10(37)$ & 0,970 \\
\hline Pressão arterial aferida na última consulta & 132 & $85,30(95)$ & $95,40(37)$ & 0,100 \\
\hline Solicitação de exame de urina no último ano & 132 & $48,90(95)$ & $77,30(37)$ & $0,003^{*}$ \\
\hline Solicitação de perfil lipídico no último ano & 132 & $63,60(95)$ & $84,10(37)$ & $0,020^{*}$ \\
\hline Altura medida em alguma consulta & 132 & $37,00(95)$ & $70,40(37)$ & $<0,001^{*}$ \\
\hline Peso aferido na última consulta & 132 & $54,50(95)$ & $81,80(37)$ & $0,010^{*}$ \\
\hline Uso de AAS & 151 & $25,40(114)$ & $32,40(37)$ & 0,490 \\
\hline \multicolumn{5}{|l|}{ Medidas de resultado } \\
\hline Grau de satisfação & 212 & $77,90(159)$ & $98,20(53)$ & $<0,010^{*}$ \\
\hline Controle pressórico & 212 & $49,00(159)$ & $44.40(53)$ & 0,510 \\
\hline Controle hemoglobina glicosilada & 38 & $47,60(23)$ & $29,40(15)$ & 0,220 \\
\hline \multicolumn{2}{|l|}{ Escore de práticas preventivas } & $\begin{array}{l}\text { Baixo Escore Geral de } \\
\text { APS Média (IC 95\%) }\end{array}$ & $\begin{array}{l}\text { Alto Escore Geral de } \\
\text { APS Média (IC 95\%) }\end{array}$ & valor-p \\
\hline & & $3,90(3,60-4,32)$ & $5,90(5,30-6,50)$ & $<0,001^{* \star}$ \\
\hline
\end{tabular}

*Significância associada ao teste de comparação de proporções utilizando a estatística $\chi^{2}$ : valor-p $<0,05$. ${ }^{*}$ Significância pelo teste $t$ para duas amostras independentes. \#Assume valores entre 0 e 10. Para todos os grupos, foram consideradas: orientação para realização de exercícios, dieta saudável, higiene bucal, pressão arterial aferida no último ano e realização de vacina contra influenza; Fumantes: orientação para cessar tabagismo; Usuários de álcool: orientação para cessar ou diminuir o hábito de beber; Diabéticos: encaminhamento para oftalmologista, encaminhamento para dentista, exame dos pés, orientação para cuidado dos pés, participação em grupo e uso de metformina; Hipertensos: participação em grupo e uso de tiazídicos; Hipertensos e diabéticos: pressão arterial aferida na última consulta, solicitação de exame comum de urina, solicitação de perfil lipídico, medida da altura e medida do peso; Indivíduos com doença cardiovascular: uso de AAS.

\section{Discussão}

O grau de orientação à APS dos diferentes tipos de serviços da rede pública de APS de Porto Alegre, medido por meio da experiência dos idosos usuários desses serviços, revelou-se muito baixo. Já a associação entre o grau de orientação à APS dos serviços públicos de APS de Porto Alegre e a qualidade do cuidado a idosos mostrou que os serviços com alto escore de APS estáo relacionados com a presença maior de práticas preventivas baseadas em evidências recomendadas a essa parcela da população.

A maior prevalência de consultas nos últimos 12 meses nos serviços da ESF e SSC-GHC demonstra que esses são serviços acessíveis à população sob sua responsabilidade. Todos os serviços apresentaram alto escore do atributo acesso-utilização e escores abaixo do ponto de corte de 6,7 para acessibilidade. A UBS, apesar de ser acessível, possui escores muito baixos em relação à coordenação e à integralidade, além de sua baixa orientação comunitária. $\mathrm{O}$ atributo coordenação do cuidado atingiu um baixo grau, ou seja, abaixo do limite para atingir o alto escore 6,7 em todos os serviços avaliados, mas ainda é melhor na ESF e no SSC-GHC. O vínculo da comunidade com seu serviço de referência é maior no SSC-GHC. A medida 
da longitudinalidade, característica necessária para o atendimento das condiçôes crônicas presentes nessa população, atinge alto grau apenas na ESF e no SSC-GHC. Esses dois serviços conseguem atender e satisfazer os pacientes com condiçôes crônicas, que não necessitam procurar outros serviços quando surgem demandas geradas por essas condições. No SSC-GHC, também é maior a proporção de usuários que referiram ter um médico de referência.

Em resumo, os escores de APS que determinam o grau de orientação à APS alcançado pelos serviços são maiores no SSC-GHC em relação à UBS e aos demais; porém, mostram-se extremamente baixos em todos os serviços. Somente o SSC-GHC atinge alto escore essencial e geral de APS. O tempo de implantaçáo maior do SSC-GHC, assim como o conhecimento sobre o serviço prestado ao longo do tempo e o sistema informatizado, podem ser fatores predisponentes ao acesso, ao vínculo maior dessas equipes com os usuários e aos melhores escores encontrados nesse serviço.

As práticas preventivas apresentaram prevalências significativamente maiores entre os usuários dos serviços de alto escore de APS, entre as quais: orientaçóes para prática de exercícios, dieta saudável, higiene bucal, referência para dentista, orientação sobre cuidados e exame anual dos pés, uso de metformina, solicitação de exame de urina e perfil lipídico, assim como medida do peso na última consulta e altura em alguma consulta. Os idosos usuários de serviços de APS com alto escore recebem melhores cuidados preventivos, como intervençôes para promover estilo de vida saudável, cuidados em saúde bucal e rastreamento de obesidade e de complicaçôes de doenças crônicas.

Apesar de não serem significativas, houve diferenças marcantes entre os escores de APS em favor do alto escore para rastreamento de hipertensão arterial e retinopatia diabética, uso de ácido acetilsalicílico (AAS) profilático, vacinação contra influenza sazonal e orientaçôes para cessação de tabagismo e cessação ou diminuição do hábito de beber. O escore de práticas preventivas é também maior nesses serviços, mostrando que o cuidado de idosos nos serviços com alto escore de APS envolve detecção de fatores de riscos, intervençôes com intuito de mudar positivamente comportamentos e controle de multimorbidades.

A natureza longitudinal do cuidado em APS proporciona múltiplas oportunidades para se realizarem intervençôes preventivas e cuidado de condiçóes crônicas. O potencial da APS no atendimento de idosos, no controle de condiçôes crônicas e na prestação de serviços preventivos é ainda pouco explorado, assim como a sua influência nos determinantes de saúde e no processo de adoecimento e dos seus efeitos na saúde da população em longo prazo. O Primary Care Medical Home (PCMH), o novo modelo de APS dos Estados Unidos, que pretende expandir o acesso e prover APS de qualidade, está positivamente associado a receber mais serviços preventivos e estar com cuidados preventivos em dia ${ }^{23}$. Os princípios do PCMH facilitam o cuidado mais oportuno, a longitudinalidade e a coordenação do cuidado e já demonstraram prestar melhor gerenciamento do cuidado das condiçôes crônicas e reduzir internaçôes desnecessárias e idas à emergência ou a serviços de pronto atendimento ${ }^{22,23}$.

Piccini et al. ${ }^{24}$ avaliaram serviços do sul e do nordeste do país quanto à provisão de APS para idosos, comparando atividades realizadas em equipes de ESF e UBS, e encontraram maior envolvimento das equipes de saúde no cuidado aos idosos e maior utilização desses serviços, medidos por meio de número de consultas, acesso a medicamentos, participação em grupos de idosos com doenças crônicas e atendimento domiciliar, nas áreas cobertas pela ESF, em comparação com a UBS ${ }^{24-27}$.

Os escores da APS, apesar de muito baixos, são maiores para idosos em relação aos alcançados quando se estudaram os adultos maiores de 18 anos de Porto Alegre. Os serviços de APS da cidade, ainda que não possam ser considerados provedores de uma APS de qualidade, estão lidando com a presença de maiores necessidades em saúde para a parcela da população idosa, o que demonstra o papel da APS como estratégia para alcançar equidade em saúde ${ }^{9,28}$. Em Curitiba, cujo sistema de saúde tem forte orientação à APS desde a década de 1970, uma avaliação feita com profissionais da saúde mostrou escores maiores que os encontrados para adultos e idosos em Porto Alegre, especialmente na ESF ${ }^{29}$. Porém, em Sáo Paulo, estudo que avaliou UBS e ESF, os escores produzidos pela avaliação de gestores e profissionais foram sempre melhores que os escores baseados na percepção dos usuários ${ }^{30}$.

O desenho de natureza transversal e a amostra pequena são limitações deste estudo, mas também podem ser explicaçôes para a significância pequena das diferenças encontradas entre os serviços. Esperar-se-ia que essas diferenças fossem maiores se fosse estudada uma amostra maior da população idosa atendida nos serviços provedores de APS da cidade. Além da temporalidade, inerente ao desenho do estudo, avaliou-se a atitude do profissional referida pelos pacientes, uma medida de processo. Não foi avaliada a adesão dos pacientes às orientaçôes e recomendaçóes como uma medida da efetividade das intervençôes, nem a modificação de desfechos. 
As informaçôes foram coletadas de acordo com o recordatório dos pacientes, sendo que o viés de memória, a idade avançada e a presença de multimorbidades também podem ser limitaçóes neste estudo. A essas limitaçóes, se somam as dificuldades para se avaliarem as atividades preventivas, cuja efetividade deve ser valorizada, principalmente em longo prazo 5 . O escore de práticas preventivas foi construído para sintetizar as informaçóes, mas não é uma escala validada, e pode também ser uma limitação. Não foi avaliado o tempo de implantação de cada tipo de serviço e de cada equipe, nem se avaliou a presença de apoio social, dois fatores que podem influenciar tanto na utilizaçáo dos serviços como na qualidade do cuidado aos idosos.

Apesar de as mudanças de comportamentos serem influenciadas por múltiplos fatores, há evidências relativas de que as recomendaçôes dadas pelos médicos têm forte influência nessas mudanças. No entanto, não há dúvidas de que a modificação do estilo de vida, por meio da combinação da diminuição do consumo de álcool, da cessaçáo do tabagismo, da prática regular de exercícios e da manutenção de uma dieta saudável, leve a uma redução da morbimortalidade ${ }^{5,13}$.

Em Porto Alegre, devido a sua especificidade e suas variadas formas de prover APS, é necessário investigar o efeito que os serviços com alto escore de APS produzem na morbimortalidade da população idosa da cidade. Pode-se também mensurar a magnitude da efetiva incorporação, à prática clínica da APS, de medidas preventivas custo-efetivas e equilibradas à prevenção oportuna.

É incompreensível que a cidade-berço da medicina de família e comunidade no país, que possui três residências na área e um serviço diferenciado na cidade para se espelhar - o SSC-GHC - apresente baixa cobertura da ESF, tenha cerca de 30\% de suas equipes permanentemente sem médicos e pobre vínculo com as comunidades, esteja investindo em Unidades de Pronto-atendimento (UPA) em vez de núcleos de apoio à saúde da família (NASF) e siga utilizando o modelo tradicional, o serviço com pior grau de orientação à APS. Isso se reflete em serviços de saúde que náo atingem escores adequados para proporcionar à populaçáo serviços de qualidade e que náo conseguem prestar adequadamente um cuidado preventivo comprovadamente eficaz.

\section{Conclusões}

Ao possuírem características fundamentais e orientadas à APS, os serviços de saúde apresentam-se mais efetivos e oferecem melhores cuidados preventivos recomendados para os idosos. Essas atividades estáo principalmente relacionadas à prevenção de doenças, como hipertensão e diabetes, condiçôes crônicas prevalentes e fatores de risco para doenças cardiovasculares, incapacidades e mortes prematuras. O sistema de saúde precisa ser remodelado para enfrentar os desafios da multimorbidade e do processo de envelhecimento. Para isso, é preciso superar o sistema fragmentado ainda vigente no Sistema Único de Saúde (SUS) e explorar o potencial da APS na gestão do cuidado dos idosos e na organização das redes de atenção.

A flexibilizaçáo da ESF, o retrocesso na conversáo da rede de APS e o fortalecimento das UPAs como porta de entrada do sistema, bem como a não incorporação de inovações e de modelos de atenção comprovadamente efetivos, são os principais obstáculos a serem enfrentados atualmente na tentativa de prover uma APS forte, integral e de qualidade. Pacientes idosos e suas famílias apresentam necessidades diferentes, que não são adequadamente satisfeitas por uma organização e uma cultura marcadas pelo atendimento equivocadamente centrado nas condições agudas e nos serviços de emergência.

\section{Referências}

1. Brasil. Ministério da Saúde. Secretaria de Atenção à Saúde. Departamento de Atenção Básica. Envelhecimento e saúde da pessoa idosa. Brasília: Ministério da Saúde; 2006. Cadernos de Atenção Básica, n. 19.

2. Brasil. Ministério da Saúde. Caderneta da saúde da pessoa idosa. Brasília: Ministério da Saúde; 2010.

3. Carvalho JAM, Garcia RA. The aging process in the Brazilian population: a demographic approach. Cad Saúde Pública 2003; 19(3): 725-33 PMid:12806475. http://dx.doi.org/10.1590/S0102-311X2003000300005

4. Instituto Brasileiro de Geografia e Estatística - IBGE. [homepage na internet]. [acesso em 29 nov 2010]. Disponível em: www.ibge.com.br

5. Haggerty J, Fortin M, Beaulieu M, Hudon C, Loignon C, Préville M, et al. At the interface of community and healthcare systems: a longitudinal cohort study on evolving health and the impact of primary healthcare from the patient's perspective. BMC Health Services Research 2010;10:258. PMid:20815880 PMCid:PMC2940881. http://dx.doi.org/10.1186/1472-6963-10-258 
6. Mendes EV. As redes de atenção à saúde. Belo Horizonte: ESP-MG; 2009.

7. Wagner EH. Chronic Disease Management: what will it take to improve care for chronic illness? Eff Clin Pract. 1998;1(1):2-4. PMid:10345255.

8. Lopéz MIV, Morata JLG, Jiménez MM, López MMV, Cavanillas AB. Intervenciones sanitarias en atención primaria que disminuyen la hospitalización por Ambulatory Care Sensitive Conditions en mayores de 65 a-os. Aten Primária 2007; 39(10): 525-34. http://dx.doi.org/10.1157/13110730

9. Starfield B. Atenção Primária: Equilíbrio entre necessidades de saúde, serviços e tecnologia. Brasília: UNESCO; Ministério da Saúde; 2002.

10. Brasil. Ministério da Saúde. Manual do instrumento de avaliação da atenção primária à saúde: primary care assessment tool pcatool - Brasil. Brasília: Ministério da Saúde; 2010.

11. Berra S, Rocha KB, Rodriguez-Sanz M, Pasarin MI, Raimil L, Borell C, et al. Properties of a short questionnaire for assessing Primary Care experiences for children in a population survey. BMC Public Health 2011; 11(285). PMid:21554717 PMCid:PMC3103459.

12. Zurro AM, Perez JFC. Atención Primaria. 6. ed. Madrid: S.A. Elsevier Espa-a; 2008.

13. Grandes G, Sanchez A, Cortada JM, Balague L, Calderon C, Arrazola A, et al. Is integration of healthy lifestyle promotion into primary care feasible? Discussion and consensus sessions between clinicians and researchers. BMC Health Serv Res. 2008; 8(213): 1-12.

14. Healthy Lives, Healthy People: Our strategy for public health in England Presented to Parliament by the Secretary of State for Health by Command of Her Majesty. 2010.

15. Harzheim E, Duncan BD, Stein AT, Cunha CRH, Gonçalves MR, Trindade TG, et al. Quality and effectiveness of different approaches to primary care delivery in Brazil. BMC Health Serv Res. 2006; 6: 156. PMid:17147819 PMCid:PMC1790713. http://dx.doi.org/10.1186/1472-6963-6-156

16. Kloetzel K, Bertoni AM, Irazoqui MC, Santos RN, Campos VGC. Controle de qualidade em atenção primária à saúde. I - A satisfação do usuário. Cad Saúde Pública 1998; 14(3): 623-8 PMid:9761617. http://dx.doi.org/10.1590/S0102-311X1998000300020

17. Demidenko E. Mixed Models Theory and Applications. New Jersey: John Wiley e Sons, Inc.; Hobokcn; 2004.

18. Riboldi J. Modelos Mistos Aplicados; $52^{\circ}$ Reunião Anual da Região Brasileira da Sociedade Internacional de Biometria; $12^{\circ}$ Simpósio de Estatística Aplicada à Experimentação Agronômica; 2007, Santa Maria. 2007.

19. Brown H, Prescott R. Applied Mixed Models in Medicine. 2nd ed. Chichester: John Wiley e Sons ed. 2006.

20. Littell RC, Milliken GA, Stroup WW, Wolfinger RD, Schanbenberger O. SAS for Mixed Models. Second ed. Cary: SAS Institute Inc; 2006. PMCid:PMC1569811

21. The GLIMMIX Procedure. SAS/STAT 9.1 User's Guide. 2011.

22. Ferrante JM, Balasubramanian BA, Hudson SV, Crabtree BF. Principles of the Patient-Centered Medical Home and Preventive Services Delivery. Ann Fam Med. 2010; 8: 108-16. PMid:20212297 PMCid:PMC2834717. http://dx.doi.org/10.1370/afm.1080

23. Pandhi N, DeVoe JE, Schumacher JR, Bartels C, Thorpe CT, Thorpe JM, et al. Preventive service gains from first contact access in the primary care home. J Am Board Fam Med. 2011; 24(4): 351-9. PMid:21737759 PMCid:PMC3137250.

24. Piccini RX, Facchini LA, Tomasi E, Thumé E, Silveira DS, Siqueira FV, et al. Necessidades de saúde comuns aos idosos: efetividade na oferta e utilização em atenção básica à saúde. Ciênc Saúde Coletiva. 2006; 11(3): 657-67. http://dx.doi.org/10.1590/S1413-81232006000300014

25. Paniz VMV, Fassa AG, Facchini LA, Bertoldi AD, Piccini RX, Tomasi E, et al. Acesso a medicamentos de uso contínuo em adultos e idosos nas regiões Sul e Nordeste do Brasil. Cad Saúde Pública. 2008 Feb; 24(2): 267-80. PMid:18278273. http://dx.doi.org/10.1590/S0102-311X2008000200005

26. Rodrigues PAM, Facchini LA, Piccinni RX, Tamasi E, Thumé E, Silveira DS, et al. Uso de serviços básicos de saúde por idosos portadores de condições crônicas. Lancet. 2009; 43(4): 604-12.

27. Thumé E, Facchini LA, Tomasi E, Vieira LAS. Home Health care for the elderly: associated factors and characteristics of access and health care. Rev Saúde Pública. 2010; 44(6): 1-9.

28. Oliveira MMC. Presença e extensão dos atributos da Atenção Primária à Saúde entre os serviços de Atenção Primária em Porto Alegre: uma análise agregada. [Dissertação]. Porto Alegre: Universidade Federal do Rio Grande do Sul; 2007.

29. Chomatas E. Avaliação da presença e extensão os atributos da Atenção Primária na rede básica de saúde no município de Curitiba. [Dissertação]. Porto Alegre: Universidade Federal do Rio Grande do Sul; 2007.

30. Elias PE, Ferreira CW, Cohn A, Kishima V, Escrivão A Jr, Gomes A, et al. Atenção Básica em Saúde: comparação entre PSF e UBS por estrato de exclusão social no município de São Paulo. Ciênc Saúde Coletiva. 2006; 11(3): 633-641. http://dx.doi.org/10.1590/S1413-81232006000300012 